OPEN ACCESS

Edited by:

Cynthia H Y Fu,

University of East London,

United Kingdom

Reviewed by:

Yosuke Morishima,

University of Bern,

Switzerland

Marco Sarchiapone,

University of Molise, Italy

${ }^{*}$ Correspondence:

Bumseok Jeong

bs.jeong@kaist.ac.kr

tThese authors have contributed equally to this work and share first authorship.

Specialty section: This article was submitted to

Computational Psychiatry, a section of the journal

Frontiers in Psychiatry

Received: 16 November 2018 Accepted: 02 April 2019

Published: 16 April 2019

Citation:

Shin DW, Yoon T and Jeong B (2019) The Associations of Emotion

Coping Appraisal With Both the

Cue-Outcome Contingency and Perceived Verbal Abuse Exposure.

Front. Psychiatry 10:250.

doi: 10.3389/fpsyt.2019.00250

\section{The Associations of Emotion Coping Appraisal With Both the Cue-Outcome Contingency and Perceived Verbal Abuse Exposure}

\author{
Dong Woo Shin ${ }^{1 \dagger}$, Taekeun Yoon ${ }^{1 \dagger}$ and Bumseok Jeong ${ }^{1,2,3 *}$ \\ ${ }^{1}$ Graduate School of Medical Science and Engineering, Korea Advanced Institute of Science and Technology (KAIST), \\ Daejeon, South Korea, ${ }^{2}$ KAIST Institute for Health Science and Technology, KAIST, Daejeon, South Korea, ${ }^{3}$ KAIST Clinic \\ Pappalardo Center, KAIST, Daejeon, South Korea
}

Previous studies have reported an association between verbal abuse in early childhood and structural and functional alterations in the young adult brain, supporting the existence of critical periods in human brain development. In addition, exposure to verbal abuse in early childhood is strongly correlated with lifetime psychiatric illness. Resilience is defined as the ability to avoid the negative psychological, biological, and social consequences of stress that impair psychological and physical homeostasis and is used to cope with these psychiatric diseases. We attempted to explain the mediatable present function of resilience and its associations with several psychiatric disorders, with verbal abuse exposure in early childhood and with the present value of the readily measurable and conceptually connected generative Bayesian model parameter. Thirty-six subjects performed a cross-modal associative learning task requiring them to learn the predictive strength of auditory cues and predict a subsequent visual stimulus. The probability of the association changed across each trial block. Subjects' responses were modeled as a hierarchical Bayesian belief-updating process using a hierarchical Gaussian filter (HGF) with three levels, a Sutton K1 model, and a Rescorla-Wagner model. Subjects completed the Korean version of the Verbal Abuse Questionnaire (VAQ) for segmented periods (aged 0 to 6,7 to 12 , and 13 to 18 years), and their positive self-appraisal was estimated using the Resilience Appraisal Scale (RAS). Random-effects Bayesian model selection identified HGF as the best model. Of the VAQ values for specific periods, only preschool VAQ scores were negatively correlated with RAS scores. The tonic volatility parameter, $\omega_{2}$, of HGF showed a negative relationship with RAS emotion coping scores. The linear regression model explained $18.3 \%$ of the variance of emotion coping appraisal with $\omega_{2}$ and preschool VAQ scores. Based on the results obtained from young adults, decrease in emotion coping appraisal can be explained by an increase in the number of experiences of verbal abuse in early childhood and the increased tendency to update beliefs about the cue-outcome associative probability in a volatile environment.

Keywords: cognitive appraisal, resilience, hierarchical Gaussian filter, verbal abuse, Bayesian learning, emotion coping appraisal 


\section{INTRODUCTION}

The core modulators of stressful experiences are predictability and controllability (1), which are associated with the uncertainty of inputs from the external world. Stress-adaptive behaviors are formed through model updates that predict and estimate uncertainty and precision-weighted prediction error. Therefore, updating the model appropriately in an uncertain environment is critical for preventing stress-induced maladaptive processes (2), which are potentially linked to stress-related disorders (3).

Many studies examining computational models of this adaptive behavior for input derived from an uncertain environment have employed physically painful feedback $(4,5)$ social stress (4), and financial reward (2). In real life, however, we mostly assess uncertainty in decision-making without painful feedback, such as electric shock, in contrast to the experimental environment. Similarly, neither a monetary reward nor social stress is a factor contributing to every decision. According to recent studies, individuals track separable forms of uncertainty sufficiently during tone-picture association learning within a nonstressful context because the uncertain environment is sufficiently stressful for the subjects $(6,7)$.

The dynamic nature of processing uncertainty in an individual varies as the organism learns about and interacts with its environment (8). Individual differences in the estimation of uncertainty might provide insights into individual resilience in a stressful environment (9). According to the cognitive appraisal theory, the quality of the emotion felt by individuals in an unforeseen environment is generated by an individual explanation of its source, feelings about the explanation, and the interpretation of the experienced arousal (10). Therefore, in the context of the emotions experienced by subjects in relation to uncertainty, individual variation in parameters estimated with computational modeling may be associated with individual differences in the adaptive process.

Resilience is a necessary skill to maintain an individual's psychological and physical condition in response to external stress (9). Moreover, resilience varies in humans, representing individual differences in an adaptive and active process in an uncertain or stressful situation $(11,12)$. Resilience may be an individual trait in that non-uniform neuroendocrine findings related to resilience have been found, and the relationship between individual resilience and psychiatric disorders such as anxiety and depression has been confirmed (9). However, little research has been performed to evaluate the relationship between the aforementioned uncertainty and individual resilience, although persons show individualized responses to uncertainty during tone-picture association learning in a nonstressful context $(6,7)$. We argue that this association needs to be studied because individual differences in model updating under stress in an uncertain situation are related to the meaning of individual resilience.

A growing body of research has begun documenting how early life stress, including emotional maltreatment, can increase vulnerability to stress-related disorders along with anatomical and functional abnormalities in the brain. The results of early stress exposure may help individuals cope with stress or increase their sensitivity to the negative effects of adult stress (13). However, excessive stress in early life exerts negative effects on changes in hippocampal structure in both animals $(13,14)$ and humans $(15,16)$. Aberrant changes in white matter structural connectivity in young adults who have experienced childhood maltreatment but do not suffer from mental disorders have also been reported in cortico-limbic or cortico-cortical connections, such as the uncinate fasciculus (17), the cingulum bundle (18), and the superior (18) and inferior (19) longitudinal fasciculus. Moreover, aberrant functional activity and connectivity have been reported in the medial frontal-amygdala circuit during the processing of contemptuous facial expressions (20), and aberrations have also been observed in ventrolateral prefrontal activity and its connectivity with the hippocampus during exposure to swear words in healthy adolescents who have been exposed to verbal abuse (21). An increase in corticotropinreleasing hormone levels in the cerebrospinal fluid of adults with a history of childhood abuse was reported (22), although this increase was not always associated with diseases such as posttraumatic stress disorder or major depressive disorder $(23,24)$.

Childhood maltreatment by caregivers is particularly considered an abuse experience that is marked by unpredictability and uncontrollability because children are not independent from their caregivers. Children who have experienced childhood abuse tend to be unable to cope appropriately with the stressful situations and tend to take a passive stance about daily stress due to failure to meet earlier developmental milestones $(25,26)$. These tendencies may lead to psychiatric problems. According to one study, parental verbal abuse at age 5 was the greatest predictor of suicidal ideation in young men with a history of childhood maltreatment (27). In addition, the odds of suicidal ideation were 2.5 times higher in young adults who had experienced sexual abuse in the preschool period than those who had had similar experiences in adolescence (28).

In particular, verbal abuse is more potently associated with scores on the psychological symptom scale than witnessing domestic violence or physical abuse (29). Additionally, verbal abuse potentially exerts a negative impact on the development of specific brain regions and increases the likelihood of developing a psychiatric disease (30). Since verbal abuse is a form of emotional maltreatment and a potent precursor of dissociation, this form of abuse has a stronger association with psychiatric sequelae than other forms of abuse $(31,32)$. Emotion coping appraisal, a component of resilience, is an important scale with which to evaluate individuals who have been verbally abused. Individuals use stimulus evaluation checks, such as a capacity for control and prediction error, to interpret external emotional stimuli, and consequently, their emotional state is adjusted by the function of emotion coping appraisal according to the cognitive theory of emotion $(33,34)$.

The probabilistic associative learning (ProAL) task enables learning and decision-making in situations where the cueoutcome contingency (cue-outcome associative probability) changes stochastically (see Figure 1A). Subjects feel that the cueoutcome contingency is uncertain when they perform the ProAL task, and this situation is the volatile environment. We tried to quantify individual differences in learning parameters under these conditions by ensuring that subjects feel uncertainty in a volatile environment. Several computational studies of decisionmaking processes have shown that subjects tune their learning rate about cue-outcome contingencies in response to a volatile 


\section{A}

\section{Changes in cue strength: ProAL}
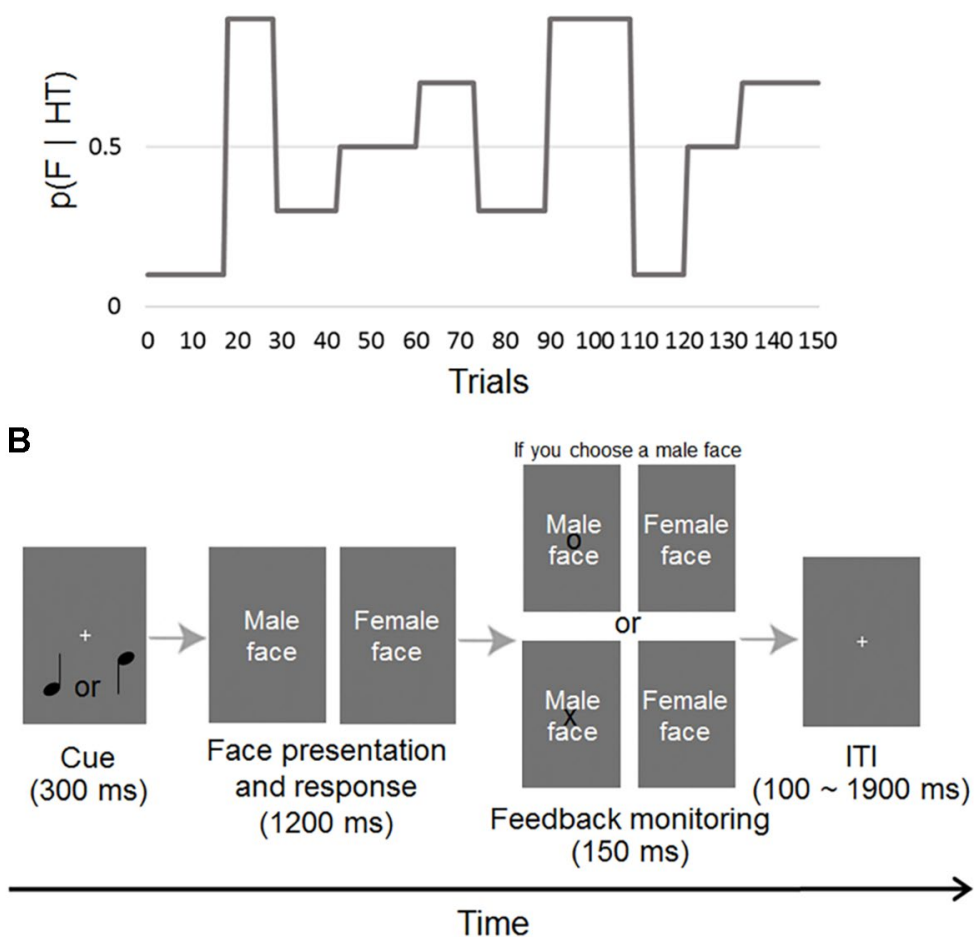

FIGURE 1 | Structure of the ProAL task. (A) The probability that a female ( $F)$ image is presented after a high tone (HT) is represented by $p(F \mid H T)$, and the change in $p(\mathrm{~F} \mid \mathrm{HT})$ over time is displayed. (B) Subjects performed a cross-modal ProAL task. This task consists of cue, face presentation and response, monitor feedback monitoring, and an ITI. This task allows the subject to learn the predictive strength of auditory cues and predict a subsequent visual stimulus. ITI, intertrial interval.

environment (35-37). However, these models are not suitable for describing learning in a volatile environment (38). Recent hierarchical models have quantified individual differences in this volatile learning $(5,6,39)$ and a hierarchical Gaussian filter (HGF, see Figure 2) with three hierarchical probability distributions is representative of various Bayesian inference models $(6,39$, 40) (see Figure 2). Research has already been conducted to identify learning-based indicators of individuals with psychiatric disorders and to identify their characteristics (38).

Based on these considerations, we hypothesized that emotion coping appraisal is correlated with HGF parameters derived from the uncertain environment and verbal abuse exposure, particularly during early childhood. If our hypothesis is validated, the "behavior" of emotion coping appraisal would be explained by the "present"

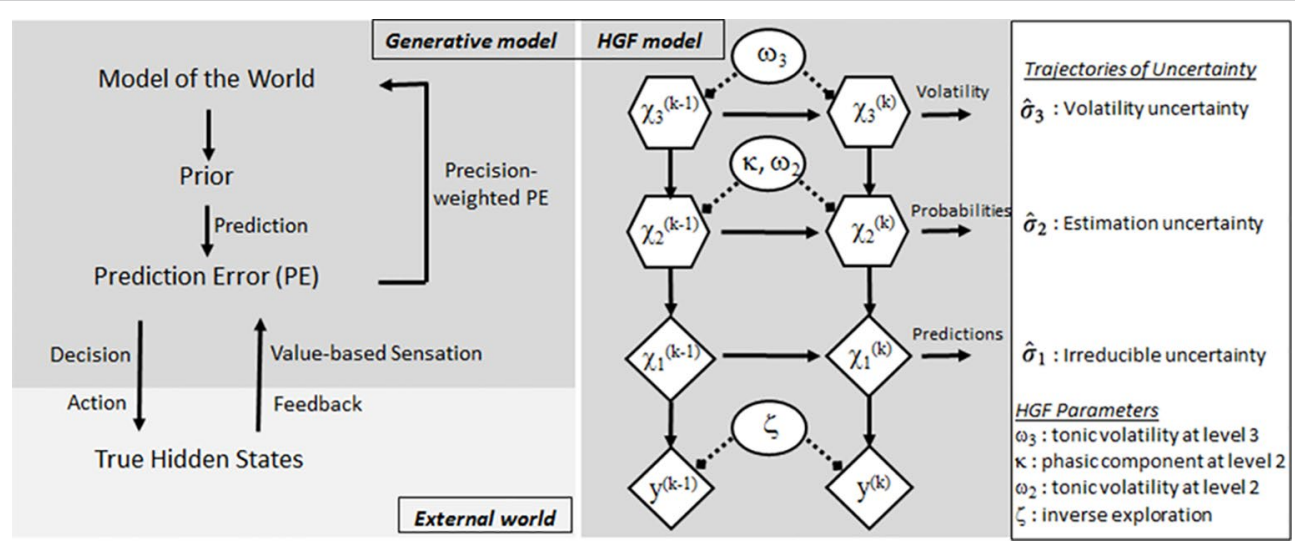

FIGURE 2 | Three-level hierarchical Gaussian filter (HGF). 


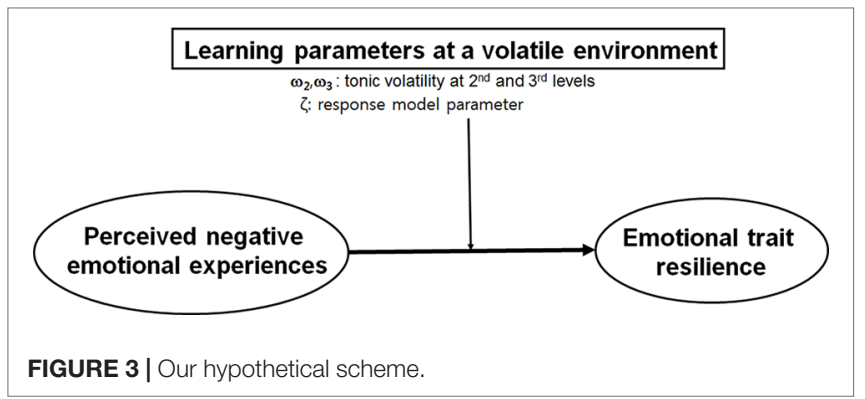

learning tendency in a volatile environment and perceived "past" verbal abuse exposure in early childhood (see Figure 3).

\section{MATERIALS AND METHODS}

\section{Subjects}

Thirty-six healthy subjects (mean age \pm standard deviation $=$ $23.4 \pm 4.1$ years, $\mathrm{M} / \mathrm{F}=23: 13$ ) participated in our experiment. The subjects were interviewed by skilled psychiatrists using the Korean version of the Diagnostic Interview for Genetic Studies (DIGS-K version 2.0) (41). The subjects did not have psychiatric or neurological illnesses, and their visual acuity or corrected vision was normal (inclusion criteria). No subjects were excluded based on the screen administered by skilled psychiatrists. Subjects were recruited from the University of Korea Advanced Institute of Science and Technology (KAIST) and provided written and informed consent. This research was approved by KAIST Institutional Review Boards (approval number: KH2013-23) and was conducted in accordance with the Declaration of Helsinki.

\section{Behavioral Task: ProAL}

The subjects performed an associative learning task in which the degree of association between two types stimuli, consisting of two auditory stimuli (low or high tones) and two visual stimuli (male and female neutral faces), changes over time. We used the human faces as stimuli in the task to study the relationship between the emotion coping ability and the learning parameters of the task. Subjects were required to constantly track the relationship over time, resulting in fluctuations in the levels of various forms of uncertainty. The probabilities governing each block varied between 90/10,70/30, and 50/50 to examine the dynamic change in uncertainty, as described in a previous study (see Figure 1A) (5). During feedback, subjects received information in the form of an "O" or " $\mathrm{X}$ " mark on the image of the face according to the correct answer (see Figure 1B). Each session of 150 trials was divided into 10 blocks of different stimulus-outcome probabilities of lengths ranging from 11 to 17 trials. The subjects performed a sufficient number of practice sessions, and we used the subsequent behavioral data obtained in the MRI scanner for analysis. A functional neuroimaging study was performed on the same subjects and the results will be published elsewhere.

\section{Behavioral Modeling}

We use three models, Rescorla-Wagner (RW), Sutton K1 (SK-1), and HGF, which are implemented in HGF module version 5.1 of Translational Neuromodeling Unit's Algorithms for Psychiatry-Advancing Science toolbox (https://www.tnu.ethz. ch/en/software/tapas.html), to model learning in the ProAL task. The HGF used here is the three-level generative model based on Bayesian inference. When a new stimulus is presented, learning at three levels of the uncertainty hierarchy occurs simultaneously with two free perceptual model parameters, $\omega_{3}$ at the third level and $\omega_{2}$ at the second level, and a response model parameter $\zeta$. The first level of HGF $\left(x_{1}\right)$ is the process of recognizing external stimuli and addresses uncertainty about outcomes (e.g., female or male image). The second level of HGF $\left(x_{2}\right)$ quantifies cue-outcome associative probability (e.g., the probability of female-high tone match) and addresses uncertainty about probabilities (cue-outcome contingencies). The third level of HGF $\left(x_{3}\right)$ represents the volatility of the probabilities (the degree of change of the second level). In other words, the third level addresses uncertainty about the environmental change. For each trial, predictions of each level occur for the next trial and are represented by a Gaussian distribution. The mean and variance of this distribution at each level are called $\hat{\mu}_{i}$ and $\hat{\sigma}_{i}$, respectively. $\hat{\sigma}_{i}$ quantifies the uncertainty of the estimate for each three level. In each stage, beliefs are updated through prediction errors. In our HGF model, the stimulus category $x_{1}$ at time $k$ is denoted by $x_{1}^{(k)} \in\{0,1\}$.

$s(\cdot)$ is a sigmoid function:

$$
s(x) \stackrel{\text { def }}{=} \frac{1}{1+\exp (-x)}
$$

The three-level hierarchy of the HGF model is described using the following equations:

$$
\begin{gathered}
x_{1}^{(\mathrm{k})} \sim \operatorname{Bernoulli}\left(s\left(x_{2}^{(k)}\right)\right) \\
x_{2}^{(k)} \sim N\left(x_{2}^{(k-1)}, \exp \left(\kappa x_{3}^{k}+\omega_{2}\right)\right) \\
x_{3}^{(k)} \sim N\left(x_{3}^{(k-1)}, \exp \left(\omega_{3}\right)\right)
\end{gathered}
$$

By definition, $\omega_{2}$ and $\omega_{3}$ represent the characteristics of subjects in the learning process for a particular situation. The value of $\omega$ represents a step size of a Gaussian random walk, and a higher value of $\omega$ is more likely to represent an unstable cue-outcome association (39). $\kappa$, the phasic component of the second-level probability distribution, was fixed. Finally, the response model was modeled using a unit square sigmoid function such that

$$
\mathrm{p}\left(y^{(k)}=1\right)=\frac{\left(\hat{\mu}_{1}^{(k)}\right)^{\zeta}}{\left(\hat{\mu}_{1}^{(k)}\right)^{\zeta}+\left(1-\hat{\mu}_{1}^{(k)}\right)^{\zeta}}
$$

where $\zeta$ is interpreted as inverse decision noise. We described the means and variance of Gaussian priors used in HGF parameter estimation in Supplementary Table 1. 


\section{MODEL VALIDATION: RANDOM-EFFECT BAYESIAN MODEL SELECTION}

The HGF was compared with SK-1 and the RW model, which is known to best explain probability-based learning among classical learning theories, to confirm that the behavioral tasks used in the experiments are appropriate to be analyzed with HGF assuming the Bayesian brain. For this comparison of the models, the random-effect Bayesian model selection (RFX-BMS) embedded in the VBA toolbox (mbb-team.github.io/VBA-toolbox/) was used $(42,43)$. We used log-model evidence approximated by the negative variational free energy under the Laplace assumption to compare the models.

\section{Clinical Variables}

The Resilience Appraisal Scale (RAS), the Korean version of Verbal Abuse Questionnaire (VAQ) to measure the perceived verbal abuse experiences, and Spielberger's State-Trait Anxiety Inventory (STAI-S/-T) were administered to subjects (see Table 1).

\section{RAS}

The RAS (44) is a 12 -item scale consisting of three 4 -item subscales estimating positive self-appraisals. These subscales focus on self-appraisals of the individual's perceived ability to cope with emotions, solve difficult situational problems, and obtain social support. An example item for assessing emotion coping appraisals is "I can handle my emotions," one for situation coping appraisals is "When faced with a problem, I can usually find a solution," and one for social support appraisals is "If I were in trouble, I know of others who would be able to help me." Responses were rated on a five-point scale from "strongly disagree" to "strongly agree." Alpha reliabilities of RAS-total ( $\alpha=$ 0.88 ), RAS-emotion coping ( $\alpha=0.92)$, RAS-situation coping ( $\alpha=$ $0.92)$, and RAS-social support $(\alpha=0.93)$ scores were significant (44). This scale has been used in young and older adults (45).

\section{Verbal Abuse Questionnaire}

Lifetime experiences of perceived verbal abuse among preschoolers (aged 0-6 years), children (aged 7-12 years), and adolescents (aged 13-18 years) were measured using the VAQ that has been validated for the Korean college population (29, 46). The VAQ is composed of 15 items covering scolding, yelling, swearing, blaming, insulting, threatening, demeaning, ridiculing, criticizing, and belittling; the perceived severity was reported using a nine-point Likert scale (from $0=$ "not at all" to $8=$ "every day") (46). Subjects reported perceived parental verbal abuse exposure at the present time. Among the clinical variables, VAQpreschool was transformed using Tukey's "ladder of powers" method (47) because of high kurtosis and skewness.

\section{Spielberger's State-Trait Anxiety Inventory}

The Korean version (48) of STAI (49), a validated questionnaire, consisted of 20 questions. The STAI-S (not at all, somewhat, moderately so, and very much so) and STAI-T (almost never, sometimes, often, and almost always) consist of a four-point scale. STAI-S indicates state anxiety and STAI-T represents trait anxiety. The scores ranged from 20 to 80 points, with higher scores indicating anxiety. Because anxiety is associated with emotion coping (50), we have measured it to confirm the indirect association with other factors using a mixed graphical model analysis.

\section{Statistical Analyses Simple Correlation Analysis}

Pearson correlation analyses were performed among HGF parameters $\left(\omega_{2}, \omega_{3}\right.$, and $\left.\zeta\right)$, RAS (total, emotion coping, situation coping, and social support appraisals), VAQ (preschool, childhood, and adolescent), STAI-S, STAI-T, and age.

\section{Linear Regression Analyses to Predict Individual's Positive Coping Appraisals}

First, we tested multicollinearity with the variance inflation factor (VIF) of a predictor variable in the following model consisting of all VAQ scores and HGF parameters:

RAS VAQ-preschool (T) + VAQ-childhood + VAQadolescent $+\omega_{2}+\omega_{3}+\zeta$

[(T): Tukey's ladder of powers transformation]

TABLE 1 | Demographic and clinical characteristics of the subjects.

\begin{tabular}{|c|c|c|c|c|c|}
\hline & Mean (SD) & Median & $\operatorname{Min} / \max$ & Skewness & Kurtosis \\
\hline Sex (male/female) & $23: 13$ & - & - & - & - \\
\hline VAQ-preschool & $5.50(10.39)$ & 3.00 & $0 / 58$ & $3.76 \ldots$ & 15.50 \\
\hline VAQ (T)-preschool & $1.31(0.98)$ & 1.47 & $0 / 4.14$ & $0.42 \ldots$ & $0.27 \ldots$ \\
\hline VAQ-childhood & $13.86(16.80)$ & 9.00 & $0.00 / 82.00$ & $6.39 \ldots$ & $2.80 \ldots$ \\
\hline VAQ-adolescent & $14.36(15.04)$ & 10.00 & $0.00 / 73.00$ & $2.06 \ldots$ & $4.76 \ldots$ \\
\hline RAS-total & $48.86(5.84)$ & 49.00 & $37.00 / 60.00$ & $-0.14 \ldots$ & $-0.67 \ldots$ \\
\hline RAS-situation coping & $16.36(2.10)$ & 16.00 & $10.00 / 20.00$ & $-0.67 \ldots$ & $1.16 \ldots$ \\
\hline STAI-S & $35.83(9.19)$ & 36.50 & $20.00 / 60.00$ & $0.47 \ldots$ & $0.08 \ldots$ \\
\hline STAI-T & $36.00(9.63)$ & 36.00 & $20.00 / 60.00$ & $0.57 \ldots$ & $-0.06 \ldots$ \\
\hline
\end{tabular}

SD, standard deviation; VAQ, Verbal Abuse Questionnaire; (T), Tukey's ladder of powers transformation; RAS, Resilience Appraisal Scale; STAI-S, Spielberger's state-trait anxiety inventory-state; STAI-T, Spielberger's state-trait anxiety inventory-trait. 
The multicollinearity test showed that high VIF values for VAQ-childhood (7.04) and VAQ-adolescent (7.01), and models for predicting RAS-total $[F(6,29)=1.458, p=0.2273]$ and subscale scores [RAS-emotion coping: $F(6,29)=2.031, p=0.0935$, RAS-social support: $F(6,29)=0.8918, p=0.5138$, RAS-situation coping: $F(6,29)=0.9834, p=0.4544]$ were not significant. If the VIF of a predictor variable was $7.04(\sqrt{ } 7.04=2.65)$, then the standard error of the coefficient of that predictor variable was 2.65 times larger than it would be if that predictor variable was not correlated with the other predictor variables. Based on results from correlation analyses and from the multicollinearity test for the models described above, we produced four different models including preschool VAQ scores (VAQ-preschool) and $\omega_{2}$, as shown below.

Simple model: RAS $\sim$ VAQ-preschool $(\mathrm{T})+\omega_{2}$

Full model: RAS $\sim$ VAQ-preschool $(\mathrm{T})+\omega_{2}+$ VAQ-preschool (T) $\times \omega_{2}$

Partial model with $\omega_{2}$ : RAS $\sim \omega_{2}+$ VAQ-preschool $(\mathrm{T}) \times \omega_{2}$

Partial model with VAQ-preschool: RAS $\sim$ VAQ-preschool $(\mathrm{T})+$ VAQ-preschool $(\mathrm{T}) \times \omega_{2}$

[VAQ-preschool $(\mathrm{T}) \times \omega_{2}$ : interaction of VAQ-preschool $(\mathrm{T})$ and $\omega_{2},(T)$ : Tukey's ladder of powers transformation]

With the exception of the "Full model" that showed multicollinearity (see Supplementary Table 2), the remaining three models were tested to predict RAS-total and subscale scores. Anxiety scores (STAI-T/-S) are not included as predictors in regression models because the tendency towards anxiety is well known to be associated with cognitive coping $(50,51)$ and because the inclusion of anxiety scores as predictors increases model complexity. The best model was selected when its adjusted $R^{2}$ had the highest value. Analysis of variance (ANOVA) was used to compare regression models. Using leave-one-out crossvalidation (LOOCV), a prediction score, $Q^{2}$, was also calculated. All statistical analyses were performed in R (version 3.5.0) (52).

\section{Mixed Graphical Model Analysis}

The role of gender should be considered in reappraisals, such as emotion regulation (53). State or trait anxiety is also related to reappraisal (51) and decision-making (54). Thus, using a mixed graphical model ("mgm" package of version 1.2-2 in $\mathrm{R} 3.5 .0$ ) for data including variables with a non-Gaussian distribution, we determined conditionally independent relationships among STAI-S or -T, gender, and predictors of the best model chosen in linear regression analyses. Here, we assume that at most, pairwise interactions exist in the true graph. The algorithm including an L1-penalty was used to obtain a sparse estimation, and the Extended Bayesian Information Criterion was applied to select the optimal regularization parameter lambda.

\section{RESULTS}

\section{Performance on the ProAL Task}

Among 150 trials, the accuracy and number of missing trials were $54.98 \pm 4.98 \%$ [mean \pm standard deviation, 45.95 (min)$64.67(\max ) \%$ ] and $0.83 \pm 1.65$ [mean \pm standard deviation, 0 ( $\min )-9(\max )]$, respectively.

\section{Behavioral Modeling}

Three models, RW, SK-1, and three-level HGF, were compared in a random-effect model (42). In RFX-BMS [Bayesian omnibus risk: $p(\mathrm{H} 0 \mid y) \geq 0.025$ ], the best model was the three-level HGF model (estimated model frequencies: 0.710, protected exceedance probabilities: 0.979; see Supplementary Table 3 and Supplementary Figure 1).

The mean values of three hierarchical learning parameters, $\omega_{2}, \omega_{3}$, and $\zeta$, in the three-level HGF model were $-5.46 \pm 2.82$, $-6.06 \pm 0.14$, and $3.16 \pm 1.94$ (means \pm standard deviations), respectively. The mean log model evidence was $-92.59 \pm 18.08$ (mean \pm standard deviation). We conducted a parameter recovery simulation to determine whether the three-level HGF model displayed internal validity. The parameters of the original model were highly correlated with the mean of recovered parameters, indicating that the parameters were reliably estimated (see Supplementary Figure 2).

\section{Correlation Analyses}

Only $\omega_{2}$ showed a significant relationship with VAQ-childhood $(r=-0.379, p=0.023)$, VAQ-adolescent $(r=-0.448, p=0.006)$, and RAS-emotion coping scores $(r=-0.333, p=0.047) . \omega_{3}$ and $\zeta$ did not show any significant relationship with clinical variables. VAQ-preschool showed a significant relationship with RAStotal $(r=-0.356, p=0.033)$ and RAS-emotion coping scores $(r=-0.361, p=0.030)$. However, VAQ-childhood and VAQadolescent scores were not correlated with RAS scores. STAI-S/-T showed a highly significant relationship with scores for RAS-total $(r=-0.506 /-0.486, p=0.002 / 0.003)$ and its subscales [social support $(r=-0.434 /-0.289, p=0.008 / 0.088)$, emotion coping $(r=-0.460 /-0.502, p=0.005 / 0.002)$, and situation coping $(r=$ $-0.422 /-0.461, p=0.010 / 0.005)]$, but not with $\omega_{2}$. In summary, $\omega_{2}$ and the VAQ-preschool score were negatively correlated with the RAS-emotion coping score, which was negatively correlated with STAI-S and STAI-T scores.

\section{Regression Analyses for Predicting the Resilience Appraisal Scale-Emotion Coping Score}

The model showing the highest adjusted $R^{2}$ value was the "Simple model" for the predicting RAS-emotion coping score (see Table 2) and the "Partial model with $\omega_{2}$ " for predicting the RAStotal score (see Supplementary Tables 4 and 5). The best model for predicting the RAS-emotion coping score explains $18.3 \%$ of the total variances in RAS-emotion coping scores (see Table 2). Cross-validation analyses showed that the "Simple model" predicted $15.6 \%$ of RAS-emotion coping scores in a new subject (see Supplementary Table 6).

\section{Mixed Graphical Model (see Figure 4)}

A mixed graphical model using an undirected graphical model with lasso depicted the associations between emotion coping and $\omega_{2}$ and VAQ in preschool and with the subject's anxiety (STAI-T/-S). The results showing the negative relationships between $\omega_{2}$ and VAQ-preschool with RAS-emotion coping were 
TABLE 2 | Multiple linear regression models using RAS-emotion coping as the dependent variable.

\begin{tabular}{|c|c|c|c|}
\hline & \multicolumn{3}{|c|}{ Dependent variable: RAS-emotion coping } \\
\hline & Simple model & Partial model with VAQ-preschool & Partial model with $\omega_{2}$ \\
\hline VAQ-preschool (T) (estimate, 95\% Cl) & $-0.891(-1.665,-0.116)^{\star}$ & $-1.938(-3.298,-0.578)^{\star \star}$ & \\
\hline$\omega_{2}$ (estimate, $\left.95 \% \mathrm{Cl}\right)$ & $-0.282(-0.550,-0.014)^{\star}$ & & $-0.445(-0.748,-0.142)^{\star *}$ \\
\hline VAQ-preschool $(T) \times \omega_{2}$ (estimate, $95 \% \mathrm{Cl}$ ) & & $-0.157(-0.330,0.016)$ & $0.119(0.008,0.231)^{*}$ \\
\hline Constant (estimate, 95\% Cl) & $15.072(13.102,17.042)^{\star \star \star}$ & $16.883(15.584,18.182)^{\star \star \star}$ & $13.851(12.195,15.507)^{\star \star \star}$ \\
\hline Adjusted $R^{2}$ & 0.183 & 0.158 & 0.168 \\
\hline Residual standard error ( $d f=33$ ) & 2.281 & 2.315 & 2.302 \\
\hline$F$ value $(\mathrm{df}=2 ; 33), p$ value & $4.923^{\star}, 0.0135^{\star}$ & $4.286^{\star}, 0.0221^{\star}$ & $4.528^{\star}, 0.0183^{\star}$ \\
\hline
\end{tabular}

RAS, Resilience Appraisal Scale; VAQ, Verbal Abuse Questionnaire; adjusted R², adjusted squared correlation coefficient; (T), Tukey's ladder of powers transformation; Cl, confidence interval; VAQ-preschool $(T) \times \omega_{2}$, interaction of VAQ-preschool and $\omega_{2}$; Simple model, RAS VAQ-preschool $(T)+\omega_{2} ;$ Partial model with VAQ-preschool, RAS VAQ-preschool $(T)+$ VAQ-preschool $(T) \times \omega_{2} ;$ Partial model with $\omega_{2}, R A S \sim \omega_{2}+$ VAQ-preschool $(T) \times \omega_{2} ;{ }^{*} p<0.05,{ }^{* *} p<0.01$, and ${ }^{* * *} p<0.001$.

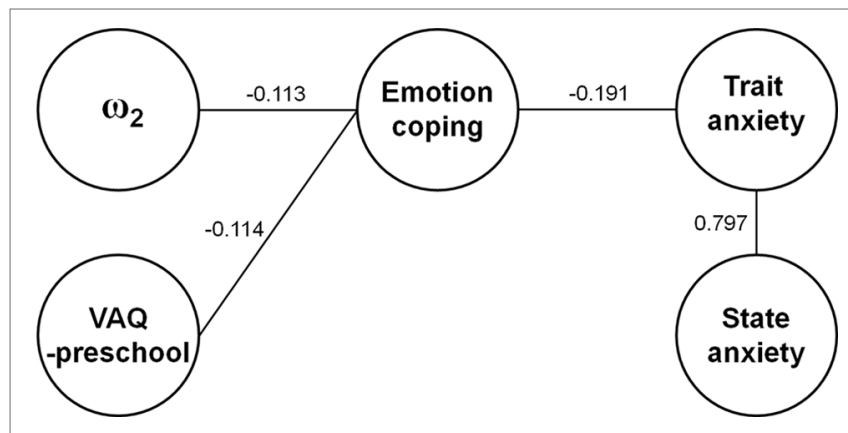

FIGURE 4 | Mixed graphical model of Resilience Appraisal Scale (RAS)emotion coping. The mixed graphical model described the correlations between the RAS-emotion coping score with $\omega_{2}$, Verbal Abuse Questionnaire (VAQ)-preschool, and trait anxiety. The gender factor was removed from the figure as it was not associated with any parameters. The estimates using the mixed graphical model function are displayed above the connecting line.

consistent with the results from the "Simple model" in regression analyses. The RAS-emotion coping score was also negatively correlated with trait anxiety (STAI-T). Gender did not have any conditionally independent relationship with other variables in the selected mixed graphical model.

\section{DISCUSSION}

In this study, using multiple linear regression analysis, we found that decreased emotion coping appraisal of resilience in young adults was explained by an increased tendency to update beliefs about cue-outcome associative probability in a volatile environment and the increase in the parental verbal abuse experienced in early childhood. The total scores of resilience appraisals were also explained in the same manner (see Supplementary Tables 4 and 7). In addition, we did not find any effect of gender on the results both with whole subject (Figure 4) and in the gender-matched subgroup (see Supplementary Figure 3).

Notably, the period in which subjects were exposed to verbal abuse was important in explaining the emotion coping appraisal of the subjects in our study. Only verbal abuse in the preschool period, but not in childhood or adolescence, was significantly associated with RAS-total and RAS-emotion coping scores in our data. Our finding is consistent with studies showing that maltreatment in early childhood is an important factor contributing to social competence associated with neuroendocrine activity (55). Additionally, childhood abuse is a very important risk factor for the development of psychopathology (56-59). The severity of the effect appears to depend on the timing of the abuse exposure $(30,60)$, and emotional abuse in early childhood, more than in other periods, is associated with an increase in the incidence of psychiatric diseases in adulthood and childhood (61).

Because resilience is defined as the ability to avoid the negative psychological, biological, and social consequences of stress that impair psychological and physical homeostasis, resilience is highly correlated across the spectrum of psychiatric diseases (12). The RAS, which estimates positive self-appraisals, is divided into emotion coping, situation coping, and social support subscales. In our study, the lower emotion coping of resilience, the greater the tendency the subject believed in cue-outcome associative probability in a volatile environment (increased $\omega_{2}$ ) in the task using social stimuli. The increased tendency to believe in cue-outcome associative probability in a volatile environment means that the degree of belief updating in each trial is high under uncertain conditions. The subjects with low emotion coping of resilience tend to fail to constantly learn about uncertain environments because the volatile environment is sufficiently stressful for the subjects. However, further studies are needed to determine whether a similar relationship exists in a ProAL task using a nonsocial association. We cautiously assume that the increased $\omega_{2}$, indicating discomposure about coping with a stressful environment, is thought to be an intermediate factor in the process of the negative emotional experience of early childhood affecting emotion coping in resilience (Figure 1). Namely, increased $\omega_{2}$ may be interpreted as a quantification of the individual trait that occurs during the process of early verbal abuse experiences affecting emotion coping in normal young adults.

Among the subscales of RAS, RAS-emotion coping reflects the mechanism regulating the effects of stress by cognitively controlling the emotional impact on the stressful environment. In addition, RAS-social support refers to solving difficult 
situational problems and RAS-social support defines the ability to obtain social support. In our study, exposure to verbal abuse and $\omega_{2}$ only explained the RAS-emotion coping score, and the scores for the remaining RAS subscales were not explained by the regression model. One potential explanation is that $\omega_{2}$, which means that model updating is affected in uncertain situations, would more likely be associated with an implied sense of emotion coping, which is the meaning of the cognitive control of emotional effects according to stressful situations, than the other two subscales of RAS.

In our mixed graphical model, "past" verbal abuse experienced in early childhood and "present" HGF learning parameters were not directly related to STAI-T (anxiety trait) but were related to RAS-emotion coping. Therefore, we are unable to eliminate the past experience, but we may reduce anxiety by improving the appraisal ability. As stress or perceived stress is potentially associated with resilience and task parameters, they should be evaluated in future studies. In addition, the HGF parameter can be used as an objective indicator of the effect of this process. The effects of the HGF parameter we suggested as a mediator requires further clinical and prospective studies.

The limitations of our study are listed below. First, this study was a cross-sectional study and was limited by the requirement to obtain recall information about verbal abuse through questionnaires. Second, although anxiety was excluded as a predictor in the regression analysis, the sample size was small compared to the variables. The mixed graphical model was used for the analysis to address this limitation. An increased sample size and cohort studies are needed. Third, the VAQ did not allow participants to reflect on the perpetrators of verbal abuse, such as peers and parents. Information about the period and perpetrators of verbal abuse would be useful, but the reliability of VAQ may decrease due to the increased number of questions. Fourth, in the case of the HGF model, the analysis of the $\kappa$ free model was excluded, and $\kappa$ was obtained by fixing $\kappa$. Fifth, we were unable to determine whether $\omega$ is a characteristic of the subject or a

\section{REFERENCES}

1. Amat J, Baratta MV, Paul E, Bland ST, Watkins LR, Maier SF. Medial prefrontal cortex determines how stressor controllability affects behavior and dorsal raphe nucleus. Nat Neurosci (2005) 8:365-71. doi: 10.1038/nn1399

2. Park H, Lee D, Chey J. Stress enhances model-free reinforcement learning only after negative outcome. PLoS One (2017) 12:e0180588. doi: 10.1371/ journal.pone.0180588

3. Brown VM, Zhu LS, Wang JM, Frueh BC, King-Casas B, Chiu PH. Associability-modulated loss learning is increased in posttraumatic stress disorder. Elife (2018) 7:e30150. doi: 10.7554/eLife.30150

4. Treadway MT, Admon R, Arulpragasam AR, Mehta M, Douglas S, Vitaliano $\mathrm{G}$, et al. Association between interleukin-6 and striatal prediction-error signals following acute stress in healthy female participants. Biol Psychiat (2017) 82:570-7. doi: 10.1016/j.biopsych.2017.02.1183

5. de Berker AO, Rutledge RB, Mathys C, Marshall L, Cross GF, Dolan RJ, et al. Computations of uncertainty mediate acute stress responses in humans. Nat Commun (2016) 7:10996. doi: 10.1038/ncomms10996

6. Iglesias S, Mathys C, Brodersen KH, Kasper L, Piccirelli M, den Ouden HE, et al. Hierarchical prediction errors in midbrain and basal forebrain during sensory learning. Neuron (2013) 80:519-30. doi: 10.1016/j.neuron.2013.09.009 parameter that changes depending on the task. Therefore, the most appropriate interpretation of $\omega$ is a characteristic parameter of the subject when he/she is performing a specific task. Sixth, we recruited subjects from a population of young people at a single university. However, the recruitment of young adults was the best way to reduce the confounding factors due to the temporal discount. Nonetheless, we should be careful to generalize our results to all ages.

The implication of our study is that the self-appraisal of individual emotion coping, a component of resilience, was explained by "the past" (verbal abuse history) and "the present" (parameters that were obtained with an objective task). Importantly, a certain period of past negative experiences had an impact on emotion coping and $\omega_{2}$ was the individually varying trait mediating the effect on behavior on coping stress of individuals who have experienced verbal abuse.

\section{AUTHOR CONTRIBUTIONS}

TY and BJ designed the study. TY collected the data. DS and BJ performed the statistical analysis. and DS, TY, and BJ wrote the first draft of the manuscript.

\section{FUNDING}

This research was supported by the Brain Research Program through the National Research Foundation of Korea (NRF) funded by the Ministry of Science \& ICT (NRF-2016M3C7A1914448 and NRF-2017M3C7A1031331).

\section{SUPPLEMENTARY MATERIAL}

The Supplementary Material for this article can be found online at: https://www.frontiersin.org/articles/10.3389/fpsyt.2019.00250/ full\#supplementary-material

7. Diaconescu AO, Mathys C, Weber LAE, Kasper L, Mauer J, Stephan KE Hierarchical prediction errors in midbrain and septum during social learning. Soc Cogn Affect Neurosci (2017) 12:618-34. doi: 10.1093/scan/ nsw171

8. Yu AJ, Dayan P. Uncertainty, neuromodulation, and attention. Neuron (2005) 46:681-92. doi: 10.1016/j.neuron.2005.04.026

9. Russo SJ, Murrough JW, Han MH, Charney DS, Nestler EJ. Neurobiology of resilience. Nat Neurosci (2012) 15:1475-84. doi: 10.1038/nn.3234

10. Colman AM. A dictionary of psychology (4th ed.). New York, NY: Oxford University Press (2015)

11. Charney DS. Psychobiological mechanisms of resilience and vulnerability: implications for successful adaptation to extreme stress. Am J Psychiatry (2004) 161:195-216. doi: 10.1176/appi.ajp.161.2.195

12. Feder A, Nestler EJ, Charney DS. Psychobiology and molecular genetics of resilience. Nat Rev Neurosci (2009) 10:446-57. doi: 10.1038/nrn2649

13. Suri D, Vaidya VA. The adaptive and maladaptive continuum of stress responses-a hippocampal perspective. Rev Neuroscience (2015) 26:415-42. doi: 10.1515/revneuro-2014-0083

14. Andersen SL, Teicher MH. Delayed effects of early stress on hippocampal development. Neuropsychopharmacology (2004) 29:1988-93. doi: 10.1038/ sj.npp. 1300528 
15. Teicher MH, Anderson CM, Polcari A. Childhood maltreatment is associated with reduced volume in the hippocampal subfields CA3, dentate gyrus, and subiculum. Proc Natl Acad Sci U S A (2012) 109:E563-E572. doi: 10.1073/ pnas. 1115396109

16. Lee SW, Yoo JH, Kim KW, Kim D, Park H, Choi J, et al. Hippocampal subfields volume reduction in high schoolers with previous verbal abuse experiences. Clin Psychopharm Neu (2018) 16:46-56. doi: 10.9758/cpn.2018.16.1.46

17. Eluvathingal TJ, Chugani HT, Behen ME, Juhasz C, Muzik O, Maqbool M, et al. Abnormal brain connectivity in children after early severe socioemotional deprivation: a diffusion tensor imaging study. Pediatrics (2006) 117:2093-100. doi: 10.1542/peds.2005-1727

18. Choi J, Jeong B, Rohan ML, Polcari AM, Teicher MH. Preliminary evidence for white matter tract abnormalities in young adults exposed to parental verbal abuse. Biol Psychiat (2009) 65:227-34. doi: 10.1016/j.biopsych.2008.06.022

19. Choi J, Jeong B, Polcari A, Rohan ML, Teicher MH. Reduced fractional anisotropy in the visual limbic pathway of young adults witnessing domestic violence in childhood. Neuroimage (2012) 59:1071-9. doi: 10.1016/j. neuroimage.2011.09.033

20. Lee SW, Yoo JH, Kim KW, Lee JS, Kim D, Park H, et al. Aberrant function of frontoamygdala circuits in adolescents with previous verbal abuse experiences. Neuropsychologica (2015) 79(A):76-85. doi: 10.1016/j. neuropsychologia.2015.10.029

21. Lee SW, Choi J, Lee JS, Yoo JH, Kim KW, Kim D, et al. Altered function of ventrolateral prefrontal cortex in adolescents with peer verbal abuse history. Psychiat Invest (2017) 14:441-51. doi: 10.4306/pi.2017.14.4.441

22. Heim C, Newport DJ, Mletzko T, Miller AH, Nemeroff CB. The link between childhood trauma and depression: insights from HPA axis studies in humans. Psychoneuroendocrinology (2008) 33:693-710. doi: 10.1016/j. psyneuen.2008.03.008

23. Yehuda R, Golier JA, Kaufman S. Circadian rhythm of salivary cortisol in holocaust survivors with and without PTSD. Am J Psychiat (2005) 162:998U5. doi: 10.1176/appi.ajp.162.5.998

24. Heim C, Newport DJ, Miller AH, Nemeroff CB. Long-term neuroendocrine effects of childhood maltreatment. JAMA (2000) 284:2321. doi: 10.1001/ jama.284.18.2317

25. Dunn EC, McLaughlin KA, Slopen N, Rosand J, Smoller JW. Developmental timing of child maltreatment and symptoms of depression and suicidal ideation in young adulthood: results from the National Longitudinal Study of Adolescent Health. Depress Anxiety (2013) 30:955-64. doi: 10.1002/ da. 22102

26. Cicchetti D, Toth SL. A developmental psychopathology perspective on child abuse and neglect. J Am Acad Child Adolesc Psychiatry (1995) 34:541-65. doi: 10.1097/00004583-199505000-00008

27. Khan A, McCormack HC, Bolger EA, McGreenery CE, Vitaliano G, Polcari A, et al. Childhood maltreatment, depression, and suicidal ideation: critical importance of parental and peer emotional abuse during developmental sensitive periods in males and females. Front Psychiatry (2015) 6:42. doi: 10.3389/fpsyt.2015.00042

28. Dunn EC, McLaughlin KA, Slopen N, Rosand J, Smoller JW. Developmental timing of child maltreatment and symptoms of depression and suicidal ideation in young adulthood: results from the national longitudinal study of adolescent health. Depress Anxiety (2013) 30:955-64. doi: 10.1002/da.22102

29. Teicher MH, Samson JA, Polcari A, McGreenery CE. Sticks, stones, and hurtful words: Relative effects of various forms of childhood maltreatment. Am J Psychiat (2006) 163:993-1000. doi: 10.1176/ajp.2006.163.6.993

30. Teicher MH, Tomoda A, Andersen SL, Neurobiological consequences of early stress and childhood maltreatment: are results from human and animal studies comparable? Ann N Y Acad Sci (2006) 1071:313-23. doi: 10.1196/ annals. 1364.024

31. Sar V, Tutkun H, Alyanak B, Bakim B, Baral I. Frequency of dissociative disorders among psychiatric outpatients in Turkey. Compr Psychiatry (2000) 41:216-22. doi: 10.1016/S0010-440X(00)90050-6

32. Teicher MH, Samson JA, Polcari A, McGreenery CE. Sticks, stones, and hurtful words: relative effects of various forms of childhood maltreatment. Am J Psychiatry (2006) 163:993-1000. doi: 10.1176/ajp.2006.163.6.993

33. Faustino AI, Oliveira GA, Oliveira RF. Linking appraisal to behavioral flexibility in animals: implications for stress research. Front Behav Neurosci (2015) 9:104. doi: 10.3389/fnbeh.2015.00104
34. Paul ES, Harding EJ, Mendl M. Measuring emotional processes in animals: the utility of a cognitive approach. Neurosci Biobehav Rev (2005) 29:469-91. doi: 10.1016/j.neubiorev.2005.01.002

35. Behrens TE, Woolrich MW, Walton ME, Rushworth MF. Learning the value of information in an uncertain world. Nat Neurosci (2007) 10:1214-21. doi: $10.1038 / \mathrm{nn} 1954$

36. Behrens TE, Hunt LT, Woolrich MW, Rushworth MF. Associative learning of social value. Nature (2008) 456:245-9. doi: 10.1038/nature07538

37. Browning M, Behrens TE, Jocham G, O’Reilly JX, Bishop SJ. Anxious individuals have difficulty learning the causal statistics of aversive environments. Nat Neurosci (2015) 18:590-6. doi: 10.1038/nn.3961

38. Lawson RP, Mathys C, Rees G. Adults with autism overestimate the volatility of the sensory environment. Nat Neurosci (2017) 20:1293-9. doi: 10.1038/nn.4615

39. Mathys CD, Lomakina EI, Daunizeau J, Iglesias S, Brodersen KH, Friston KJ, et al. Uncertainty in perception and the hierarchical Gaussian filter. Front Hum Neurosci (2014) 8:825. doi: 10.3389/fnhum.2014.00825

40. Mathys C, Daunizeau J, Friston KJ, Stephan KE. A Bayesian foundation for individual learning under uncertainty. Front Hum Neurosci (2011) 5:39. doi: 10.3389/fnhum.2011.00039

41. Joo EJ, Joo YH, Hong JP, Hwang S, Maeng SJ, Han JH, et al. Korean version of the diagnostic interview for genetic studies: validity and reliability. Compr Psychiatry (2004) 45:225-9. doi: 10.1016/j.comppsych.2004.02.007

42. Stephan KE, Penny WD, Daunizeau J, Moran RJ, Friston KJ. Bayesian model selection for group studies. Neuroimage (2009) 46:1004-17. doi: 10.1016/j. neuroimage.2009.03.025

43. Rigoux L, Stephan KE, Friston KJ, Daunizeau J. Bayesian model selection for group studies-revisited. Neuroimage (2014) 84:971-85. doi: 10.1016/j. neuroimage.2013.08.065

44. Johnson J, Gooding PA, Wood AM, Tarrier N. Resilience as positive coping appraisals: testing the schematic appraisals model of suicide (SAMS). Behav Res Ther (2010) 48:179-86. doi: 10.1016/j.brat.2009.10.007

45. Gooding PA, Hurst A, Johnson J, Tarrier N. Psychological resilience in young and older adults. Int J Geriatr Psych (2012) 27:262-70. doi: 10.1002/gps.2712

46. Jeong B, Lee SW, Lee JS, Yoo JH, Kim KW, Cho S, et al. The psychometric properties of the Korean version of the Verbal Abuse Questionnaire in university students. Psychiat Invest (2015) 12:190-6. doi: 10.4306/pi.2015.12.2.190

47. Tukey JW. Exploratory data analysis. Boston, Massachusetts, USA: Reading, Mass (1977).

48. Hahn DW, Lee CH, Chon KK. Korean adaptation of Spielberger's STAI (K-STAI). Korean J Health Psychol (1996) 1:1-14.

49. Spielberger CD, Gorsouh RL. Manual for the state-trait anxiety inventory. Palo Alto, California: Consulting Psychologists Press (1970).

50. Legerstee JS, Garnefski N, Jellesma FC, Verhulst FC, Utens EM. Cognitive coping and childhood anxiety disorders. Eur Child Adolesc Psychiatry (2010) 19:143-50. doi: 10.1007/s00787-009-0051-6

51. Goldin PR, Manber-Ball T, Werner K, Heimberg R, Gross JJ. Neural mechanisms of cognitive reappraisal of negative self-beliefs in social anxiety disorder. Biol Psychiatry (2009) 66:1091-9. doi: 10.1016/j.biopsych.2009.07.014

52. $\mathrm{R}$ Core Team $R$ : A language and environment for statistical computing. Vienna, Austria: R Foundation for Statistical Computing (2017).

53. Nolen-Hoeksema S. Emotion regulation and psychopathology: the role of gender. Annu Rev Clin Psychol (2012) 8:161-87. doi: 10.1146/ annurev-clinpsy-032511-143109

54. Hartley CA, Phelps EA. Anxiety and decision-making. Biol Psychiatry (2012) 72:113-8. doi: 10.1016/j.biopsych.2011.12.027

55. Hart J, Gunnar M, Cicchetti D. Salivary cortisol in maltreated children: evidence of relations between neuroendocrine activity and social competence. Dev Psychopathol (1995) 7:11-26. doi: 10.1017/S0954579400006313

56. Edwards VJ, Holden GW, Felitti VJ, Anda RF. Relationship between multiple forms of childhood maltreatment and adult mental health in community respondents: results from the adverse childhood experiences study. Am J Psychiatry (2003) 160:1453-60. doi: 10.1176/appi.ajp.160.8.1453

57. Kendler KS, Kuhn JW, Prescott CA. Childhood sexual abuse, stressful life events and risk for major depression in women. Psychol Med (2004) 34:147582. doi: $10.1017 /$ S003329170400265X

58. Post RM, Leverich GS, Xing G, Weiss RB. Developmental vulnerabilities to the onset and course of bipolar disorder. Dev Psychopathol (2001) 13:581-98. doi: 10.1017/S0954579401003091 
59. Sansone RA, Sansone LA, Wiederman M. The prevalence of trauma and its relationship to borderline personality symptoms and self-destructive behaviors in a primary care setting. Arch Fam Med (1995) 4:439-42. doi: 10.1001/archfami.4.5.439

60. De Bellis MD, Keshavan MS, Clark DB, Casey BJ, Giedd JN, Boring AM, et al. Developmental traumatology. Part II: brain development. Biol Psychiatry (1999) 45:1271-84. doi: 10.1016/S0006-3223(99)00045-1

61. Gilbert R, Kemp A, Thoburn J, Sidebotham P, Radford L, Glaser D, et al. Recognising and responding to child maltreatment. Lancet (2009) 373:16780. doi: 10.1016/S0140-6736(08)61707-9
Conflict of Interest Statement: The authors have neither financial nor nonfinancial competing interests to declare.

Copyright (c) 2019 Shin, Yoon and Jeong. This is an open-access article distributed under the terms of the Creative Commons Attribution License (CC BY). The use, distribution or reproduction in other forums is permitted, provided the original author(s) and the copyright owner(s) are credited and that the original publication in this journal is cited, in accordance with accepted academic practice. No use, distribution or reproduction is permitted which does not comply with these terms. 